\title{
La stanza di Giorgio Manganelli
}

\section{Elisabetta Orsini}

Università degli Studi di Roma «Tor Vergata»

pelasgi7@alice.it

\begin{abstract}
L'avversione di Giorgio Manganelli nei confronti del concetto d'autore era connessa a un ideale polimorfico e cangiante dell'individualità, a una critica dell'io, inteso come struttura stabile, riconoscibile, rappresentabile. Le sue concezioni filosofiche, lo spingevano a considerare l'arte come un processo impersonale. Lo psicanalista junghiano Ernst Bernhard gli aveva insegnato che lo spazio della memoria può dilatarsi oltre l'insieme dei ricordi del singolo individuo, per comprendere differenti regimi di oggetti come ad esempio il mondo animale, vegetale e minerale. Manganelli usava la scrittura per entrare in questo lungo repertorio di variazioni. Una delle sue immagini preferite era quella dell'uomo che si trasforma in un calamaio di inchiostro e che intinge il pennino in sé medesimo per poter scrivere delle cose, quasi trasformandosi in esse.
\end{abstract}

Parole chiave: autore, scrittura, luogo di lavoro, Roland Barthes, Gilles Deleuze.

\begin{abstract}
Giorgio Manganelli's aversion to the concept of the author was linked to a polymorphic and changing ideal of individuality, to a critique of the self, conceived as a stable, recognisable and representable structure. His philosophical ideas drove him to consider art as an impersonal process. Ernst Bernhardh, the Jungian psychoanalyst, had taught him that memory space can expand beyond memories of every individual, to understand different patterns of objects, for example the animal world, the plant world and the mineral world. Manganelli used writing to enter into this long repertoire of variations. One of his favourite images was that of a man transforming into an inkwell who wets the nib in himself in order to write things, thereby becoming these things.
\end{abstract}

Key words: author, writing, work place, Roland Barthes, Gilles Deleuze. 


\section{Introduzione - L'autore non esiste}

Scrivendo su Manganelli, mi torna in mente, come un monito, una frase del filosofo Gilles Deleuze: "Quando scrivo su un autore, il mio ideale sarebbe di riuscire a non dire nulla che possa rattristarlo». ${ }^{1}$ Infatti sembra impossibile scrivere su Manganelli senza pensare di doverlo rattristare e contraddire. Se si parla della sua attività di scrittore, s'incorre necessariamente in quello che, ai suoi occhi, sarebbe apparso come un duplice errore: in primo luogo s'inventa una soggettività, poi le si attribuisce la responsabilità dell'artificio letterario, creando la figura altrettanto fittizia dell'autore. In questo modo si realizza la mistificazione del soggetto-autore, verso la quale Manganelli nutriva la massima avversione.

Dunque per scrivere di Manganelli e del suo spazio di lavoro, vorrei partire proprio da tale avversione. La sua antipatia nei confronti del concetto d'autore era connessa a un ideale polimorfico e cangiante dell'individualità, a una critica dell'io, inteso come struttura stabile, riconoscibile, rappresentabile. Le sue concezioni filosofiche, lo spingevano a considerare l'arte come un processo impersonale. Un processo che sempre supera l'apposizione di una soggettività creativa, che sempre la disconosce, e che può edificare la propria necessaria forza terapeutica soltanto su tale spietato disconoscimento.

Credo che Manganelli possa essere associato a Gilles Deleuze, con il quale condivideva - come vedremo - un concetto clinico della scrittura. Ma a fianco di Manganelli si potrebbe anche sentire la presenza di Roland Barthes. Come Barthes, Manganelli avrebbe potuto scrivere di sé:

Egli sopporta male qualsiasi immagine di se stesso, soffre a venir nominato. Considera che la perfezione d'un rapporto umano è basata su questo vuoto d'immagine... ${ }^{2}$

Quale può essere il viatico della scrittura se non questo vuoto d'immagine? L'individuo è una sorta di ricettacolo di contraddizioni non eludibili, non ricomponibili e quindi la prima tappa verso la scrittura è il disconoscimento e superamento del feticcio dell'io. Scrive nei suoi quaderni: "Questo è il punto: (...) frantumare l'io in una pluralità di oggetti». ${ }^{3}$

1. G. Deleuze, C. Parnet, Dialogues, Paris: Flammarion, 1996. Trad. del red.

2. R. BARTHES, Barthes di Roland Barthes, Torino: Einaudi, 1980, p. 51.

3. G. Manganelli, Quaderno 10.3.1951-2.11.1952, p. 102, cit. in M. CaVADINI, La luce nera, Milano: Bompiani, 1997, p. 35. Manganelli non riconosceva l'esistenza di una personalità unitaria, ma parlava di un io frammentato e molteplice, spesso contraddittorio. Dichiarava esplicitamente in un'intervista: «A quel tempo, in fondo, avevo un'idea coerente di me. Avevo l'idea che avrei dovuto essere un'unica immagine coerente. Avere un'unica autobiografia (...) la prima cosa che ha provocato in me l'impatto con Bernhard [Ernst Bernhard, n.d.r] è stato proprio rompere quell'idea lì. L'idea dell'unicità dell'io e quindi una decomposizione dell'immagine della mia personalità, di quello che io ero. Questa è stata la prima cosa che ho capito e che non mi ha più abbandonato. Questa scoperta l'ho fatta mia». [C. CARDONA, «Da un'autobiografia all'altra. Intervista a Manganelli» in Immediati 
Uno dei presupposti del suo lavoro, è questa frantumazione: «Ciascuno di noi non è un io, è un noì. ${ }^{4}$ Deve essere immediatamente superata anche la convinzione che esista un autore, un soggetto di scrittura, il soggetto di una enunciazione espressiva. Questo soggetto d'enunciazione esiste soltanto nel caso della comunicazione e non in quello della scrittura vera e propria. Il soggetto e l'autore costituiscono lo stadio regressivo della scrittura, appartengono ai casi della rappresentazione autobiografica, che nulla ha a che vedere con l'arte. Per parlare di Manganelli, bisogna dunque partire da questa sua volontà di svanire ed estinguersi sul foglio. Di sostituire la propria scomoda corporeità fisica con la sostanza egualmente fisica, materiale della letteratura: il foglio, l'inchiostro.

\section{Frantùmati!}

La perdita di sé è la condizione primaria affinché si possa scrivere: anzi la scrittura è esattamente una perdita di sé, un'abrasione dell'io. Senza questo processo di oltrepassamento della soggettività, non esiste la scrittura ma piuttosto qualcosa che le somiglia (la comunicazione, la divulgazione...):

L'acquisizione dell'anonimato è necessaria per l'accesso al luogo della parola, del terrore, dell'assenza, dell'invenzione, delle origini (...) Se l'abrasione del nome viene consumata, la parola ci priva di anima, nel caso ne avessimo una, e di tutto ciò che ci sta dentro (...) Quando siamo vuoti e anonimi, la parola può cominciare ad agire, purché abbiamo la cortesia, naturalmente, di toglierci prima la pelle. ${ }^{5}$

Si tratta di un processo di regressione a uno stato dell'essere non informe, ma poliforme o piuttosto trasformativo.

Superare l'io, vuol dire accedere alla propria autentica condizione di essere frantumato, contraddittorio, continuamente difforme da sé, in perenne trasformazione. Scrivendo si accede alla linea viva che unisce questi oggetti frantumati, queste pluralità disperse. Si comincia a entrare in questi frammenti. Scrivere è quindi tacere l'io: parlare le parole del linguaggio, le parole d'altre cose e di altri esseri cui si partecipa e che addirittura si è, qualora si superino i confini ristretti della soggettività.

dintorni, 1990, p. 209]. In Hilarotragoedia troviamo la teoria degli eidola, delle infinite animule che compongono l'uomo: «'io non è da vedersi nella somma di codesti eidola, né strettamente parlando, nelle loro relazioni: ma in ciascuno d'essi; e se accade, come accade, che io creda di essere più l'uno che l'altro di quelli, ciò viene dal prevalere o decadere di uno o altro eidolon: ma io sono pur sempre tutte, e ciascuna, di codeste animule (...) essendo io ciascuno di quegli eidola, codesti destini reciprocamente repugnanti mi appartenevano integralmente. Ciò generava furori interiori, disperazioni, deliri, euforie, frodi, violenze e diserzioni» [G. Manganell, Hilarotragoedia, Milano: Adelphi, 1987, p. 74].

4. ID., Il vescovo e il ciarlatano, Roma: Quiritta, 2001, p. 86.

5. ID., Discorso dell'ombra e dello stemma, Milano: Rizzoli, 1982, p. 64. 


\section{Dice Roland Barthes:}

Scrivere implica necessariamente tacere; scrivere è in un certo modo, farsi muto come un morto, diventare uno a cui non è consentita l'ultima replica; scrivere è dal primo momento offrire all'altro quest'ultima replica. ${ }^{6}$

Il monito di Manganelli deve essere preso assolutamente sul serio: frantùmati!...:

Gli animali nascono dai sassi; come hai chiuso in te la bambola, ora chiuderai e schiuderai la bestia. Frantùmati: non aver paura della tua scomparsa; non puoi perderti, solo frantumarti una volta e una volta; così continui. Sasso, la notte abdica; sosta e rompiti in vita. ${ }^{7}$

Il pericolo che Roland Barthes aveva individuato era quello dell'aggettivazione. Barthes, come Manganelli, voleva porre al centro del processo di scrittura non l'artista-autore dell'opera, bensì una specie di vuoto, che con forza centrifuga organizzasse intorno al suo vortice, l'intero universo delle parole. Dice Manganelli in un'intervista:

Nuovo Commento è nato proprio come un problema tecnico che mi interessava particolarmente: costruire un libro intorno ad una «assenza», un "vuoto»... Il non libro centrale costituisce una specie di gorgo di niente intorno a cui si aggrega il libro, per cui il libro è disposto tutto intorno al suo centro; ma questo luogo che non c'è, è in realtà la parte attiva del libro, è la parte che regge tutta la struttura del racconto, del testo... ${ }^{8}$

Il vuoto centrale di questo testo corrisponde esattamente al vuoto dell'autore, che è struttura fittizia in Barthes come in Manganelli. Scrive Barthes in Critica e verità:

Il soggetto non è una pienezza individuale che si ha il diritto di evacuare nel linguaggio, ma viceversa un vuoto attorno al quale lo scrittore intreccia una parola infinitamente trasformata (inserita in una catena di trasformazioni), cosicché ogni scrittura che non mente designa l'assenza del soggetto anziché i suoi attributi interiori. Il linguaggio non è il predicato di un soggetto inesprimibile, o che il linguaggio stesso servirebbe a esprimere, ma è il soggetto (...) A dar luogo al simbolo è la necessità di designare instancabilmente il nulla dell'io che io sono. ${ }^{\prime}$

Sappiamo che Manganelli era un lettore appassionato dei testi di Roland Barthes con il quale condivideva importanti punti di vista. In primo luogo l'attribuzione di una soggettività alla scrittura, al linguaggio stesso che diviene, in entrambi, il vero protagonista dell'opera. Scrive Roland Barthes:

6. R. Barthes, Saggi critici, Torino: Einaudi, 1966, p. XIII.

7. G. Manganelli, Dall'inferno, Milano: Adelphi 1998, p. 50.

8. ID., La penombra mentale, Interviste e conversazioni 1965-1990, Roma: Editori Riuniti, 2001, p. 52.

9. R. BARTHES, Critica e verità, Torino: Einaudi, 1969, p. 57-58. 
Scrivere per frammenti: i frammenti sono allora delle pietre sulla circonferenza del cerchio: mi sparpaglio in tondo: tutto il mio piccolo universo a pezzi; al centro cosa? ${ }^{10}$

Scrive Manganelli:

Noi portiamo nel diaframma un'arnia di caverne, cavità orifizi (...) e lì appunto si collocano elettivamente le parole. ${ }^{11}$

Così come il commento si organizza intorno a un testo vuoto, simile ad una grotta («un centro minimo, ma potentemente elastico, carico di oscure, intense implicazioni; una grotta; l'appunto di una necropoli; un'arcaica, sigillata tomba» ${ }^{12}$ ), l'io è una cavità o un anfratto in cui ci si può unicamente perdere. Il linguaggio diviene il vero soggetto della scrittura, mentre lo scrittore diventa una sua semplice funzione.

\section{Il rumore sottile della prosa}

Un'opera importante dovrebbe essere considerata priva d'autore, anonima. Figlia unicamente della letteratura stessa. Le opere di Kafka e di Dostoevskij sono state scritte da personaggi puramente inventati, creature della fantasia:

...quanto più è grande uno scrittore, più anonimo diventa. Il suo nome serve solo per essere conosciuto in società. Borges e Kafka sono due insiemi di parole. $^{13}$

Una posizione volutamente paradossale ma che rivela il modo in cui Manganelli pensava la scrittura, la letteratura e quindi il metodo di lavoro.

La scrittura è un'arte dell'abbandono e - allo stesso tempo — un'arte del più acuto controllo. Lo scrittore accetta di divenire lo strumento della lingua, ma soltanto per trasformarsi nel suo più perfetto esecutore. Non sa dove le sue parole lo condurranno, lo ignora in anticipo, ma questo non significa che, nel momento in cui scrive, non padroneggi il percorso irrefrenabile che - condotto allo stesso tempo da se stesso e da altro - può scegliere di realizzare.

Chi scrive è più simile a un medium o a uno sciamano che a un letterato: è un folle e un alchimista piuttosto che un intellettuale impegnato. Per la sua definizione, occorre fare riferimento a queste figure, che condensano le qualità e le caratteristiche dell'uomo che scrive: il fool, lo sciamano, l'alchimista. ${ }^{14}$ Occorre utilizzare un vocabolario denso di termini apparentemente contra-

10. ID., Barthes di Roland Barthes, cit., p.107.

11. G. MANGANELli, Discorso dell'ombra e dello stemma, cit., p. 98.

12. ID., Nuovo commento, Torino: Einaudi, 1969, p. 75-76.

13. G. MANGANELLI, La penombra mentale, cit., p. 207.

14. Più avanti torneremo su questa specie di archetipi mitologici dello scrittore, figure assai ricorrenti negli scritti di Manganelli. 
stanti, perché lo scrittore domina ed è dominato; è attivo e passivo; sognatore e sognato; sa perdersi e - contemporaneamente- esercitare il più energico controllo; è capace di abbandonarsi e di essere in uno stato d'assoluta tensione. È una persona che si muove con abilità tra ciò che conosce e quanto ignora, tra la veglia e il sonno e così via.

Così come il medium riesce a parlare la lingua del fantasma abolendo la propria voce e la propria identità, lo scrittore trascrive le voci che sente risuonare dentro la cavità vuota di se stesso, parla la voce del morto:

Odo voci, e sebbene sappia che esse vengono da esseri vivi, so anche che esse vengono dall'abitacolo del mio corpo, vengono, sommesse ma pronte a farsi urlo, dalle mie viscere, le pagine interne, che leggono se stesse a gran voce. Io devo trascrivere le voci, perché io non sono uomo, ma un vecchio castello affollato di fantasmi. ${ }^{15}$

Chi scrive non sa cosa fa: lo stato di passiva-attività in cui viene a trovarsi durante la scrittura gli permette una sorta di controllo spontaneo del linguaggio, un controllo a-volontaristico e interiorizzato delle parole. Dalla prima riga fino all'ultima, scrive sotto dettatura, ignorando dove andrà a finire:

Il cosiddetto scrittore è coinvolto in una tensione specifica con una struttura linguistica e questo coinvolgimento è lo scrivere. Ben difficile sarebbe dire che colui che scrive sia un autore, giacché egli non sa esattamente a che cosa stia attendendo, quale sia la destinazione di quello che fa e non sa nemmeno, mentre scrive la prima riga, quale sarà l'ultima del testo... Ad un certo momento, egli è uno che scrive sotto dettatura. ${ }^{16}$

Un'immagine che si trova anche in Marguerite Yourcenar, che considerava lo scrittore un medium, un trascrittore di voci provenienti da altri mondi, interiorizzate attraverso lo studio approfondito ed erudito della storia:

Un personaggio creato da noi non muore più (...) si fa tacere completamente il proprio pensiero, si ascolta una voce (...) E una presenza quasi materiale, si tratta per così dire, di una visitazione (...) In definitiva, lo scrittore è il segretario di se stesso. Quando scrivo, porto a termine un compito, sono in qualche modo, sotto il mio stesso dettato; compio il lavoro faticoso e difficile di ordinare il mio stesso pensiero, il mio dettato. ${ }^{17}$

Manganelli sottolineava che lo scrittore «non sa» anche per criticare delle idee di letteratura che considerava estremamente avvilenti.

17. M. Yourcenar, Ad occhi aperti - Conversazioni con Matthieu Galey, Milano: Bompiani, 1982, p. 137 e 208. 
La letteratura non serve a esprimere delle idee perché se così fosse lo scrittore non sarebbe una persona che sa inoltrarsi nelle zone notturne e inesplorate del suo animo, bensì un intellettuale, un comunicatore. La letteratura non è trasmissione di idee, altrimenti possederebbe dei contenuti e decadrebbe dalla sua condizione sacrale, mitologica: «le idee sono deboli e con le idee non si fa letteratura (...) Detesto le idee. Le idee servono solo ai giornalisti». ${ }^{18} \mathrm{Si}$ scrive in uno stato d'incosciente equilibrismo linguistico:

Quando si dice che uno scrittore è cosciente di ciò che fa, si enuncia una contraddizione. Quando uno parla non dice quello che pensa; uno capisce quello che pensa, ascoltandosi. ${ }^{19}$

Tra i personaggi cui Manganelli ama associare lo scrittore, quello del fool, più di ogni altro, rivendica la sua idea di una scrittura di niente. Uomo di corte, il fool è dedito all'arte della menzogna: straparla, scioccheggia, fabula e affabula ma gli ripugnano le idee, le verità, almeno quelle dichiarate, quelle rivelate.

Manganelli esalta la letteratura come menzogna. Non è un caso che abbia sottolineato, nella sua copia dei Saggi critici di Barthes, il seguente passaggio:

...il solo compito dell'artista è di esplorare tutte le significazioni possibili, ciascuna delle quali, presa a sé, sarà solo menzogna (necessaria), ma la cui molteplicità sarà la verità stessa dell'artista. Ecco il paradosso (...): dire la verità è mentire. ${ }^{20}$

Manganelli è pienamente d'accordo con Barthes e Blanchot quando afferma in un quaderno: «La poesia dice nulla: è̀. ${ }^{21}$ In questo anticipa addirittura le parole di Blanchot, che ne Lo spazio letterario scrive:

La letteratura è. Ciò che essa dice è esclusivamente questo: che essa è -e niente di più. Al di fuori di questo, non è niente. Chi vorrebbe che esprimesse qualcosa di più, non trova niente, trova che essa non esprime niente. ${ }^{22}$

18. G. Manganelli, La penombra mentale, cit., p. 207.

19. Ibidem. Parole che ricordano quelle di Merleau-Ponty: «Le mie parole sorprendono me stesso e mi insegnano il mio pensiero" [M. Merleau-Ponty, Problemes actuels de la Phénoménologie, Bruxelles: 1952, p. 97. Trad. del red.]. Dichiara ancora Manganelli in un'intervista: "A me pare evidente che impariamo quello che sappiamo quando lo diciamo. Quando io parlo so quello che penso. Non è il contrario (...) La parola ha qualcosa da dire a colui che parla (...) Io, per sapere cosa penso, devo parlare (...) Quando scrivo è addirittura diabolico. È un correre dietro continuamente alle parole. Le parole sono sempre più avanti, sono sempre adescanti ed elusive» [G. MANGANELLI, La penombra mentale, cit., p. 193194]. Manganelli ha sempre nutrito un forte legame con la filosofia francese. La sua idea della scrittura non trova tanti punti di contatto, analogie, nell'entourage degli scrittori italiani, mentre risulta sempre molto affine a quella di questi pensatori.

20. R. BARTHES, Saggi critici, Torino: Einaudi, 1966, p. 200.

21. G. ManganelLI, Quaderno. Appunti critici 1948-49, par. 32, p. 28, cit. in M. CaVAdinI, La luce nera, cit. p. 12.

22. M. Blanchot, Lo spazio letterario, Torino: Einaudi, 1975, p. 8. 
Da questo punto di vista, Barthes, Blanchot e Manganelli sono molto affini. Tutti e tre celebrano la letteratura come menzogna, per rintracciare proprio nel retaggio della menzogna una possibilità di verità, l'unica possibilità di verità, anche se si tratta di una verità ambigua ed enigmatica. Del resto sembra che la menzogna letteraria (oscura, sibillina, onirica) abbia uno stile molto simile a quello della complessa-oscura verità e che soltanto lo scrittore possa inoltrarsi in questi territori senza smarrirsi. Scrive Blanchot:

Ciò che colpisce nella letteratura è che l'inganno e la mistificazione non soltanto sono inevitabili, ma formano l'onestà dello scrittore, la parte di speranza e di verità che è in lui. ${ }^{23}$

Manganelli è più provocatorio di Blanchot; non dimentica mai di essere un narratore e, anche nei suoi saggi più eruditi, ironizza come un fool. Viceversa, nei suoi libri di narrativa assume il tono serio di un saggista e inventa intere mitologie per spiegare il significato della scrittura.

La menzogna letteraria rappresenta una possibilità di salvezza. Manganelli lo racconta con il mito di Moloch, inserito nel Discorso dell'ombra e dello stemma. Moloch è il signore e padrone del mondo: una sorta di mostro sacrificale, non molto distinto dal mondo stesso, l'uno e l'altro essendo caratterizzati da uno stesso senso di distruzione e di annientamento di sé medesimi. Moloch è dedito ai sacrifici umani. Solo attraverso questi sacrifici il mondo ha modo di salvarsi e dunque di continuare a distruggersi e morire. Un sistema feroce e molto collaudato. Un ragazzo che nasca in questo mondo non ha grandi possibilità: può diventare egli stesso un ministro del male e dunque un assassino, un adoratore del fuoco e della ferocia, oppure può sfuggire al proprio assassino gettandosi da solo nel fuoco e dunque suicidandosi:

....sia che si uccida, sia che uccida, egli non può uscire dal regno angusto e decisivo di quell'essenza centrale, il signor Fuoco, e un fuoco, si noti, che chiama a sé ma non concede luce. ${ }^{24}$

Ma si apre uno spiraglio: la possibilità di fuggire stando fermi ed evitando tanto l'assassinio quanto il suicidio. Bisogna iniziare a parlare, diventare uno stregone del centro del mondo e coinvolgere il centro negativo della macchinazione, in un incantesimo. Adescare e irretire Moloch con le parole. Il ragazzo che scelga questa strada deve parlare ininterrottamente e dimenticare il proprio nome in funzione di questa sua totale trasformazione:

quando egli era uomo di Moloch, come suicida o omicida, egli sapeva tutto di sé, giacché il fuoco e il nulla sono altamente didascalici; ma dal momento

23. M. BlanCHOT, La follia del giorno-La letteratura e il diritto alla morte, Reggio Emilia: Elitropia, 1982, p. 76.

24. G. Manganelli, Discorso dell'ombra e dello stemma, cit., p. 53. 
che ha scelto di parlare con Moloch, a Moloch, di Moloch, egli non sa più nulla di sé, è tutto parole, incantesimi, magherie, nonsense, rime, allitterazioni, ossimori, velari, palatali, e la sua occulta volontà lessicale e sintattica lo guida, gli fiata nell'intimo, e il mondo esiste, sebbene Moloch non si nutra, sebbene il nulla centrale sia sempre al suo posto, ma la sua sordità eterna è stata intaccata. ${ }^{25}$

Le parole sclerotizzano e affievoliscono il potere del male. Anche l'approssimarsi del momento della morte, diventa per lo scrittore un'esperienza innocua e necessaria, quella di una morte per delega, totalmente accidentale. Il ragazzo-scrittore

deve ininterrottamente parlare; oltre, oltre; e procedere verso il corpo abitato dal corpo furibondo; e non sa, o teme di sapere, che anche a lui toccherà, come era nel suo destino originario, entrare nel fuoco, ma che parlando egli esegue anche un incantesimo su se medesimo, egli sarà nel fuoco, ma vi sarà come l'uomo dello specchio e le fiamme insieme lo bruceranno orrendamente e gli saranno innocue e necessarie; egli entrerà nel fuoco non per perirvi ma per delega, giacché egli è il qualcuno del tutto anonimo, scelto aprendo a caso la guida del telefono, che deve occuparsi delle parole. ${ }^{26}$

Ritorna sempre il tema dell'anonimato, come la scelta fondamentale di chi voglia scrivere. Una delle immagini preferite da Manganelli era quella dell'uomo che si trasforma in un calamaio di inchiostro e che intinge il pennino in sé stesso: per scrivere delle cose ma quasi trasformandosi in esse, attraverso l'imitazione o meglio l'evocazione che possono farne i segni. È fondamentale abbandonare il proprio nome, distruggerlo alla radice, per poter divenire pennino, inchiostro, animale, pianta, architettura barocca...

Ernst Bernhard gli aveva insegnato che lo spazio della memoria può dilatarsi oltre l'insieme dei ricordi del singolo individuo, per comprendere differenti regimi di oggetti: il mondo animale, vegetale, minerale. ${ }^{27}$ Manganelli usava la scrittura per entrare in questo lungo repertorio di variazioni. Per lui era impossibile vivere senza la letteratura, prescindendo da questa esperienza.

\section{Manganelli e Deleuze}

Questo breve capitolo è scritto a titolo di esperimento. In verità non ho mai letto niente che facesse minimamente ipotizzare un rapporto tra Manganelli e il filosofo Gilles Deleuze. Scrivo per effetto di una suggestione. La suggestione crea-

25. Ibidem, p. 54.

26. Ibidem.

27. Ernst Bernhard è l'analista junghiano che ha curato Manganelli. Vedi a questo proposito: E. Trevi, Come si diventa uno scrittore: lo spazio psichico di Giorgio Manganelli, in Giorgio MANGANELLI, Il vescovo e il ciarlatano, cit., p. 100-101. 
ta dalla sottile affinità che mi è sembrato di scorgere tra il pensatore francese e lo scrittore italiano. Somiglianza che mi piacerebbe riassumere in tre principali nodi tematici: il primo è il presupposto dell'anonimato e dell'impersonalità; il secondo è l'idea di una letteratura intesa come un divenire; il terzo è il valore clinico-terapeutico della scrittura.

$\grave{E}$ difficile poter parlare una terminologia che appartenga a Deleuze come a Manganelli, senza privilegiare un vocabolario piuttosto che un altro e quindi senza assimilare impropriamente i due autori. Azzarderò comunque questa falsificazione, anche in omaggio a Manganelli che scriveva: «L'unica vostra speranza è nella citazione errata». 28

Per quanto riguarda il concetto d'impersonalità, questo era per Deleuze - come per Manganelli-il necessario punto di avvio della sperimentazione. Manganelli usa il termine "conoscenza» e scrive: "Il conosci te stesso dei greci è un imperativo ironico: significa perditi in te stesso». ${ }^{29} \mathrm{Il}$ superamento della soggettività è per entrambi la condizione imprescindibile della scrittura. Una scrittura che è anche sperimentazione, liberazione e salvezza.

La scrittura desoggettivante deleuziana, così come la scrittura desoggettivante manganelliana sono egualmente due forme anarchiche - addirittura terroristiche - di liberazione dell'individuo da un male non soltanto esistenziale ma anche sociale. Ne La letteratura come menzogna e in altri piccoli saggi, Manganelli ribadisce il valore asociale e anarchico della letteratura: un esplosivo inesauribile, frutto dell'opera minuziosa e ardita di un essere inutile e pericoloso come lo scrittore (associato comunque al matto): «Non v'è letteratura senza diserzione, disubbidienza, indifferenza, rifiuto dell'anima». ${ }^{30} \mathrm{La}$ scrittura è salvezza in quanto forma di liberazione dal Moloch (male sociale e ferocia naturale della vita) e dal carcere dell'Io. Le due cose sono comunque strettamente connesse: il Moloch e la soggettivazione-assoggettante che questi impone. Il Moloch e l'io.

L'individualità dovrebbe avere confini più vasti: siamo molteplici, siamo (per Deleuze) evenemenziali, pure compossibilità di eventi nello spettro, accidentale, di un'ecceità. L'individualità dovrebbe avere confini più vasti: siamo un'infinità di eidola, di animucule distinte, conflittuali e spesso in lotta tra loro (Manganelli), che si ampliano ad altri fondamentali influssi. Secondo Manganelli siamo moltissime cose (soprattutto quando scriviamo): piante, animali di tutti i tipi, enti geometrici, oggetti geologici e planetari (stelle, comete, pianeti...). Chi ha praticato la scrittura di Manganelli sa benissimo che queste esperienze non costituiscono l'eccezione ma la regola costante della sua narrativa.

L'io ha confini vastissimi quando scrive: Manganelli e Deleuze sono perfettamente d'accordo su questo e per entrambi i limiti di una destratificazione 
della soggettività (Deleuze) o di un'abrasione dell'io (Manganelli) sono decisi soltanto da una sorta di prudenza. Scrive Manganelli:

...solo chi è tanto disadattato da poter diventare anonimo può sperimentare una condizione di creatività impersonale. Psicologicamente, tutto ciò comporta un rischio, come ogni volta che l'Io cosciente deve essere invaso ma non predato, ferito ma non leso. ${ }^{31}$

Scrive Deleuze:

Non bisogna procedere a colpi di martello, ma con una lima molto fine (...) Disfare l'organismo non ha mai voluto dire uccidersi, ma aprire il corpo a connessioni che suppongono tutto un concatenamento, circuiti, congiunzioni, suddivisioni e soglie, passaggi e distribuzioni d'intensità, territori e deterritorializzazioni misurate alla maniera di un agrimensore (...) Al limite, disfare l'organismo non è più difficile che disfare gli altri strati, significanza e soggettivazione. (...) L'arte comune delle tre operazioni è la prudenza; e se capita che si rasenti la morte disfacendo l'organismo, quando ci si sottrae alla significanza e all'assoggettamento si rasenta il falso, l'illusorio, l'allucinatorio, la morte psichica. ${ }^{32}$

In Deleuze l'impercettibilità è la strategia di fuga della prigione dell'io: «essere impercettibile è la condizione o il risultato di uno sforzo per disfare la vita della forma generica o personale che la imprigiona». ${ }^{33} \mathrm{Ma}$, — sostiene Deleuze- «E' ben difficile riuscire a essere completamente sconosciuto, sconosciuto anche alla propria portinaia, o nel proprio quartiere». ${ }^{34}$ Bisogna acquisire una sobrietà inglese, l'understatement:

E' necessaria molta ascesi, molta sobrietà e involuzione creatrice: un'eleganza inglese, un tessuto inglese, confondersi con i muri, eliminare il troppo visto, il troppo da vedere (...) tutto quel che fa mettere radici a ciascuno (a tutti) in se stesso. 35

In Manganelli è l'anonimato dello scrittore a garantire l'esperienza della scrittura:

Lo scrivere non è professione dell'Io. Attinge a zone anonime della persona. Collodi non è l'autore di Pinocchio. È portatore di una funzione che ignora: poter vivere la genialità pur restando un borghesuccio di Pescia. ${ }^{36}$

31. G. MANGANelli, Il vescovo e il ciarlatano, cit., p. 64.

32. G. Deleuze, F. Guattari, Mille piani, Roma: Istituto dell'Enciclopedia Italiana, 1987, v. I, p. 232-233.

33. P. Mengue, Gilles Deleuze ou le système du multiple, Paris: Editions Kimé, 1994, p. 290. Trad. d. red.

34. G. Deleuze, C. Parnet, Dialogues, cit., p. 57.

35. G. Deleuze, F. Guattari, Mille piani, cit., v. I, p. 406.

36. G. MANGANELli, La penombra mentale, cit., p. 40. 


\section{Dichiara ancora Manganelli:}

Tutti i libri sono punti interrogativi. La letteratura non può mai essere autobiografica. Io sono convinto che l'autore non esiste, esiste una distesa di macerie fantastiche e questa si organizza in un testo all'apparenza coerente, ma non diventa mai un luogo in cui noi ci possiamo collocare. Anche le macerie sono un modo di atteggiarsi dell'enigma. ${ }^{37}$

La letteratura nasce proprio su tale sconfessione del nome:

Lo scrittore, lui, è un pover'uomo; è una funzione sindacale in un senso molto umile: non gli è neppure necessario avere un nome. Non è un caso che noi abbiamo dei libri straordinari che non hanno autore. Ė sconvolgente e meraviglioso che la letteratura europea cominci con dei libri senza autore, semplici edifici di parole tra cui l'autore stesso è stato distrutto, rimpiazzato da un nome valido per chiunque. Ogni autore è fondamentalmente, sostanzialmente, anonimo... ${ }^{38}$

Essere anonimi ed essere impercettibili sono due modalità differenti di una identica sparizione: lo scrittore diventa una linea astratta o una pura funzione dello scrivere. In Manganelli la caverna dell'io completamente vuota, diventata un piccolo tabernacolo d'inchiostro e il pennino s'intinge in questa sostanza fluida dell'anima trascinandola nei segni. E impossibile non percepire questo movimento estremamente fluido:

Non stupisca dunque il lettore se (...) l'uomo dei commenti (...) immerga codesto comprimario da avanspettacolo, nel lubrico calamaio della bocca, nel suo bavoso inchiostro, i gialli calami delle dita e con speciose interpunzioni di denti, dilati sul foglio una malsana, inquinata nota in calce. ${ }^{39}$

\section{E ancora:}

Tutto ho scritto intingendo la spennata penna del mio ingegno nel mio negrissimo inchiostro interiore; così come gli uomini compilano i propri sogni, intingendosi, pennini di se medesimi, nel calamaio della notte. ${ }^{40}$

La scrittura è fluida e mescola le cose trascinandole in un inarrestabile processo di trasformazione. Ecco un piccolo frammento di scrittura manganelliana:

Dagli orecchi escono foglie di acanto, che si intrecciano ai cardi della lingua di lui e non v'è su tutti i corpi peluria che non diventi labirinto, graffito, incisione, invenzione, capitello, tutto ciò che di contorto può attorcersi ad un essere alfabetico. ${ }^{41}$ 
Manganelli era un grande amante del barocco (figurativo e letterario), proprio come Deleuze. In Manganelli il Barocco è addirittura il prototipo della letteratura, nel senso che Manganelli estende all'intera letteratura alcune caratteristiche peculiari dell'arte barocca (ricchezza, opulenza, arte della piega e della reiterazione):

L'oggetto letterario è oscuro, denso, direi pingue, opaco, fitto di pieghe casuali, muta costantemente linee di frattura, è una taciturna trama di sonore parole. ${ }^{42}$

\section{Scrive Deleuze:}

Il Barocco non rimanda a un'essenza, ma piuttosto a una funzione, a un tratto. Non smette mai di fare pieghe (...) il barocco avvolge e riavvolge le pieghe, le spinge all'infinito, piega su piega, piega secondo piega. ${ }^{43}$

È la scrittura che permette, in Deleuze, l'accesso al divenire delle cose, la sperimentazione:

scrivere è un divenire, scrivere è un'attraversata di divenire che non sono divenire-scrittore, ma divenire-topo, divenire-insetto, divenire-lupo... ${ }^{44}$

\section{Scrive Deleuze in Critica e clinica:}

Divenire non significa raggiungere una forma (identificazione, imitazione, Mimesis), ma trovare la zona di vicinanza, d'indiscernibilità o d'indifferenziazione tale da non potersi più distinguere da una donna, da un animale $\mathrm{o}$ da una molecola. ${ }^{45}$

Deleuze parla molto del divenire animali e curiosamente i testi manganelliani sono stracolmi di personaggi che divengono animali: completamente o soltanto parzialmente. Talvolta non si capisce se si tratti di uomini o di animali, tanto forte sembra in Manganelli quella zona di vicinanza e di indiscernibilità, di cui parlava Deleuze.

Scrive Manganelli:

Dal ratto decomposto escono innumeri piccoli ratti, si guardano attorno, si dirigono verso di me. Ormai tutto è buio, io prendo a correre, mi accorgo di essere un animale velocissimo, passo fra i frantumi dei vetri, corro, corro, oh il rumore minuto dei passi che mi inseguono! Mi seggo ci sediamo nell'anfiteatro di una spaziosa aula, siamo decine di diligenti discepoli, prendiamo appunti, qualcuno ha il registratore, girano foglietti. La lezione promette di essere impor-

42. ID., La letteratura come menzogna, cit., p. 176.

43. G. Deleuze, La piega, Torino: Einaudi, 1990, p. 5.

44. G. Deleuze, F. Guattari, Mille piani, cit., v. I, p. 348.

45. G. Deleuze, Critica e clinica, Milano: Raffaello Cortina Editore, 1997, p. 20. 
tante. Guardo la cattedra, e vedo a questa mollemente appoggiato l'anfesibena. Dunque l'anfesibena, l'anfesibena, l'animale che va avanti e indietro, insegna all'università, sebbene ignori di quale sorta di università si tratta. So di essere due persone, un giovane studioso e un assassino inseguito dai ratti, ma mi pare ragionevole non scegliere fra le due vocazioni. ${ }^{46}$

Manganelli era ossessionato dagli animali. A questo proposito, non si può dimenticare la curiosa testimonianza di Pietro Citati, che ricordando l'amico scomparso, scrive:

Assomigliava a un animale: un animale uscito da casa a caccia di cibo, insidiato da altri animali feroci e pericolosi, che si nascondevano chissà dove. Una volta un nostro amico comune disse che Manganelli non era antropomorfo; e lui si divertì molto. Aveva sempre saputo di essere della razza di Gregor Samsa. Aveva sempre saputo che uno scrittore, se vuole discendere nell'immensa zona desolata, dove si annidano le ombre dell'inconscio e dell'essere, deve diventare animale: un ratto, un cane, un malinconico tapiro. ${ }^{47}$

Manganelli è riuscito a trasformarsi in un personaggio di romanzo, ovvero in un animale manganelliano (un malinconico tapiro). Sembra che sia riuscito nell'impresa tentata da Roland Barthes in Barthes di Roland Barthes. Barthes invitava a considerare il suo scritto autobiografico come un'invenzione romanzesca e premetteva alla sua opera la frase in esergo: "Tutto ciò deve essere considerato come detto da un personaggio di romanzo». ${ }^{48} \mathrm{E}$ inoltre sorprendente che Citati per raccontare la scrittura di Manganelli, parli proprio di un divenire animale: utilizzando, forse inconsapevolmente, la terminologia di Gilles Deleuze.

\section{Luogo di lavoro}

Uno stanzino, oppure anche un palcoscenico. Queste sono le due curiose definizioni che Manganelli ha dato del suo spazio di scrittura. In un certo senso, in queste definizioni, si condensano alcune sue idee intorno allo scrivere. Stanzino e palcoscenico sono due modi di definire la condizione contraddittoria di chi scrive. Lo scrittore è una persona che per scrivere degnamente, deve astrarsi dal mondo e ritirarsi in uno spazio isolato, simile a una cella monacale.

Ma lo scrittore è anche un attore, un esibizionista mendace, menzognero, anarchico, sopraffatto dall'obbligo di falsificare, il cui spazio è quello del palcoscenico.

Il luogo di lavoro reale di Manganelli era ovviamente soltanto lo stanzino, in cui si ritirava a scrivere per giorni e notti, ininterrottamente. Ma quella del palcoscenico è stata un'interessante metafora, introdotta in un piccolo scritto intitolato proprio Luogo di lavoro. Un saggio provocatorio, in cui la scrittura 
viene celebrata come una rappresentazione scenica e teatrale, condotta con gesti poveri e ripetitivi:

Sto scrivendo il testo che a qualcuno accadrà di leggere; e mi accorgo che questo mio scrivere non è, propriamente, scrivere, ma eseguire gesti e movimenti, variamente ritmati, in uno spazio delimitato; questo spazio poi dovrebbe, anzi lessicalmente è la mia scrivania, immersa nel consueto spaurito disordine, in una caotica vessazione; ma sarà bene che io mi renda conto che non tanto di scrivania si tratta, ma di palcoscenico, di spazio scenico, di luogo deputato ad eventi sostanzialmente teatrali, il teatro del lavoro. ${ }^{49}$

Gli altri tipi di lavoratori sembrano maggiormente consapevoli del carattere puramente rappresentativo dei loro gesti: lo scrittore è convinto della veridicità dei suoi movimenti ed è ignaro della loro vis teatrale:

Mentre colui che scrive è convinto in effetti di essere intento a scrivere, mentre è intento alla recita di scrivere, chi panifica, misura, pesca, mura edifici e scialba muri, una qualche segreta fantasia che quel che fa sia rappresentazione non può fare a meno di averla (...) dubbi ve ne sono verso colui che scrive, perché i suoi gesti sono poveri e ripetitivi, perché solo di rado si muove a cercare in appositi cataloghi una qualche parola, o magari uno spunto in un libro, tutti gesti che, fatti una volta, saranno sempre identici, e a dire il vero monotoni assai e anche deprimenti. ${ }^{50}$

Il luogo di lavoro è lo spazio di una gestualità teatrale, rappresentativa. Potrebbe essere un luogo piccolo e stretto come uno stanzino, purché vi si possa ogni volta riprodurre la cerimonia della scrittura. La scrivania è un piccolo palcoscenico, in cui i gesti dello scrittore sono quelli irruenti e allo stesso tempo cauti di chi deve mantenere alta la tensione, riuscendo a esercitare un grande autocontrollo:

Il cosiddetto scrittore è coinvolto in una tensione specifica con una struttura linguistica e questo coinvolgimento è lo scrivere (...) Ricordo che un critico come Alain diceva: Se si sbaglia una parola, non si corregge, si getta la pagina. Quell'errore significa che si è perso il contatto con la tensione. ${ }^{51}$

Lo studio è lo spazio, eminentemente mentale, in cui lo scrittore si abbandona alla scrittura come a un precipizio calcolato. ${ }^{52}$ Non è un luogo fisico, ma uno stato della mente, e sussiste ovunque lo scrittore riesca a realizzare la ten-

49. G. Manganelli, Il rumore sottile della prosa, Milano: Adelphi, 1994, p. 15.

50. Ibidem, p. 16 e 17.

51. G. MANGANELli, La penombra mentale, cit., p. 54 e 216.

52. «Noi crediamo di vivere, quando il nostro compito sarebbe quello di accadere. Ma l'accadere, come dice anche il verbo che è molto bello, implica questo abbandono, implica questa specie di precipizio calcolato in cui noi ci depositiamo, se siamo capaci di farlo" [G. MANGANELLI, La penombra mentale, cit., p. 67]. 
sione e la concentrazione necessarie alla sua scrittura. La tensione non deve conoscere attimi di sospensione, fino al compimento dell'opera. Pena il fallimento dell'opera stessa, che si costruisce unicamente attraverso quest'impresa:

È terribile quando mi metto a scrivere: sono come un funambolo, avanzo, non so più dove sono - a metà? a due terzi? - e arrivato alla fine, cado (...) non sono un vero scrittore: un vero scrittore non ha paura di scrivere, di avanzare... ${ }^{53}$

Manganelli evoca sempre panorami di vita cinquecenteschi: quelli della corte, delle fucine alchemiche, o della strada. Vi sono giullari, scribacchini, scienziati alchimisti, acrobati e saltimbanchi, e lo scrittore è una sintesi di tutte queste figure, oppure è, volta per volta, ciascuna di esse. Condivide con giullari, acrobati e alchimisti la cura estrema del particolare, la ricerca di un perfezionismo meticoloso e attento (anche quando non esibito): virtuosismo linguistico del buffone, vigile ponderazione delle sostanze nell'alchimista, arte dell'equilibrio nel funambolo.

Nel suo luogo di lavoro aleggiano queste figure di riferimento, con le quali l'artista s'identifica. Emblemi della scrittura letteraria, come anche il personaggio di Pinocchio, verso il quale Manganelli nutriva una passione particolare e cui ha dedicato un libro: Pinocchio un libro parallelo. Alcuni grandi pinocchi di legno erano posti sulla sua scrivania, come numi protettori di una letteratura fondata sulla menzogna. A proposito della passione per i pinocchi e della loro presenza nello studio, un intervistatore racconta:

Un Pinocchio enorme, classicamente vestito di rosso e verde domina la scrivania seguito a breve distanza da altri due esemplari di media e bassa statura, mentre altri sono sparsi più in là: negli angoli della casa, inaccessibili. "Quello lì, il più grande -Manganelli lo indica con particolare amore- l'ho comprato io quando sono stato in pellegrinaggio a Collodi, gli altri me li hanno tutti regalati e vivono qui con me». ${ }^{4}$

Manganelli scriveva chiuso nel suo studio (quando inseguiva un'idea era capace di restarci per dieci giorni e dieci notti), scherzosamente definito «deposito di carta igienica ${ }^{55}$, fino a che, sfinito, terminava il suo scritto. Soltanto allora poteva cadere in terra, come un medium che venga improvvisamente abbandonato dal suo fantasma: «... arrivato alla fine, cado». ${ }^{56}$

A questo punto, lo scrittore poteva finalmente disconoscersi:

Sono estremamente disorientato all'idea che io scriva dei libri e che questi vengano pubblicati e presi sul serio (...) Ho l'impressione che tutto sia una burla. ${ }^{57}$

53. Ibidem, p. 136.

54. Ibidem, p. 109.

55. Così presentava il suo studio a un giornalista: «questo è il mio stanzino da lavoro, detto deposito di carta igienica» [G. MANGANELLI, La penombra mentale, cit., p. 120].

56. Ibidem, p. 136.

57. Ibidem, p. 32. 